\title{
Reversal of brain aging by targeting telomerase: A nutraceutical approach
}

\author{
DIMITRIS TSOUKALAS ${ }^{1-3^{*}}$, ANA MARIA BUGA $^{4 *}$, ANCA OANA DOCEA $^{5 *}$, EVANGELIA SARANDI $^{2,6}$, \\ RADU MITRUT $^{7}$, ELISAVET RENIERI ${ }^{6,8}$, DEMETRIOS A. SPANDIDOS $^{9}$, ION ROGOVEANU $^{10}$, \\ LILIANA CERCELARU $^{11}$, MIHAELA NICULESCU ${ }^{11}$, ARISTIDIS TSATSAKIS ${ }^{6}$ and DANIELA CALINA ${ }^{1}$ \\ ${ }^{1}$ Department of Clinical Pharmacy, University of Medicine and Pharmacy of Craiova, 200349 Craiova, Romania; \\ ${ }^{2}$ Metabolomic Medicine, Health Clinic for Autoimmune and Chronic Diseases, 10674 Athens, Greece; \\ ${ }^{3}$ European Institute of Nutritional Medicine (E.I.Nu.M.), I-00198 Rome, Italy; Departments of ${ }^{4}$ Biochemistry and \\ ${ }^{5}$ Toxicology, University of Medicine and Pharmacy of Craiova, 200349 Craiova, Romania; ${ }^{6}$ Laboratory of Toxicology and \\ Forensic Sciences, Medical School, University of Crete, 71003 Heraklion, Greece; 7 Department of Cardiology, \\ University and Emergency Hospital, 050098 Bucharest, Romania; ${ }^{8}$ Environmental Engineering Laboratory, Department of \\ Chemical Engineering, Aristotle University of Thessaloniki, 54124 Thessaloniki; ${ }^{9}$ Laboratory of Clinical Virology, \\ Medical School, University of Crete, 71409 Heraklion, Greece; Departments of ${ }^{10}$ Internal Medicine and \\ ${ }^{11}$ Anatomy and Embryology, University of Medicine and Pharmacy of Craiova, 200349 Craiova, Romania
}

Received 22 July 2021; Accepted September 7, 2021

DOI: $10.3892 /$ ijmm.2021.5032

\begin{abstract}
Telomeres, the protective caps of chromosomes, shorten with age, as telomerase, the enzyme responsible for the compensation of telomere erosion, is inactive in the majority of cells. Telomere shortening and subsequent cell senescence lead to tissue aging and age-related diseases. Neurodegenerative disorders, characterized by the progressive loss of neurons among other hallmarks of aged tissue, and poor cognitive function, have been associated with a short telomere length. Thus, telomerase activity has emerged as a therapeutic target, with novel agents being under investigation. The present study aimed to examine the effects of a novel natural telomerase activator, 'Reverse ${ }^{\mathrm{TM}}$ ', containing Centella asiatica extract, vitamin C, zinc and vitamin D3 on the brains of 18-month-old rats. The administration of the 'Reverse ${ }^{\mathrm{TM}}$, supplement for 3 months restored telomerase reverse transcriptase (TERT) expression in the brains of rats, as revealed
\end{abstract}

Correspondence to: Professor Anca Oana Docea, Department of Toxicology, University of Medicine and Pharmacy of Craiova, 2 Petru Rareș Street, 200349 Craiova, Romania

E-mail: ancadocea@gmail.com

Professor Daniela Calina, Department of Clinical Pharmacy, University of Medicine and Pharmacy of Craiova, 2 Petru Rareș Street, 200349 Craiova, Romania

E-mail: calinadaniela@gmail.com

*Contributed equally

Key words: telomerase activators, telomerase reverse transcriptase, aging, Centella asiatica, vitamin C, zinc, vitamin D by ELISA and immunohistochemistry. In addition, the findings from PCR-ELISA demonstrated an enhanced telomerase activity in the cerebellum and cortex cells in the brains of rats treated with the 'Reverse ${ }^{\mathrm{TM}}$ ' supplement. The histopathological findings confirmed a structural reversibility effect close to the differentiation observed in the young control group of rats treated with two capsules/kg body weight of the 'Reverse ${ }^{\text {TM }}$, supplement. On the whole, the findings of the present study provide a strong indication that an increased telomerase activity and TERT expression may be achieved not only in the postnatal or embryonic period, but also in the brains of middle-aged rats through nutraceutical supplementation. The use of the 'Reverse ${ }^{\mathrm{TM}}$ ' supplement may thus contribute to the potential alleviation of a number of central nervous system diseases.

\section{Introduction}

Telomerase is a ribonucleoprotein enzyme which functions as a reverse transcriptase to maintain the length of telomeres, by adding tandem short-sequence repeats at the end of chromosomes, thus compensating for the loss of deoxyribonucleic acid (DNA) during genome replication. It consists of the protein telomerase reverse transcriptase (TERT) and the telomerase RNA component (TERC), which facilitate telomere synthesis and is regulated by several proteins that are bound to TERT and TERC, forming the enzymatically active telomerase (1). In addition, TERT has been reported to perform additional to telomere elongation functions, including its translocation to the mitochondria and its contribution to the decrease in oxidative stress-related complications upon the accumulation of reactive oxygen species (ROS) (2). It has been demonstrated that upon increased levels of oxidative stress or other less common stimuli, TERT is located in the mitochondria where 
it reduces mitochondrial, as well as nuclear DNA damage (3), enhances the natural antioxidant and detoxification cellular mechanisms (4) and improves respiratory chain function, thereby reducing ROS production $(5,6)$. Telomerase is inactive or expressed at low levels in the majority of somatic cells; however, an increased activity is observed in highly proliferating cells, such as adult stem cells, male sperm cells, activated lymphocytes and some epidermal cells $(7,8)$. Consequently, somatic cells have a definite number of divisions, upon which they become senescent or undergo apoptosis through a mechanism that involves telomeres and the DNA damage response (9). Briefly, telomeres are DNA sequences at the very end of chromosomal DNA consisting of the TTAGGG pattern, bound to the shelterin proteins, forming a DNA-protective structure known as the T-loop (10). This structure protects the coding DNA sequences lying before the telomeres from exonuclease activity or DNA damaging agents (11). However, telomere length decreases with each cell replication by several hundred bases due to the inability of DNA polymerase to replicate the $3^{\prime}$ telomeres and maintain their length to the daughter DNA strands. Telomeres shorten gradually up to a critical point at which they signal cell proliferation arrest to prevent cellular and DNA damage (12). Senescent or dying cells are replaced by somatic stem cells of the respective tissue to maintain tissue function. The dominant hypothesis of aging and age-related diseases suggests that the shortening of the telomeres of somatic stem cells hampers their tissue renewal capacity, which leads to the accumulation of senescent cells in the tissue and, respectively, to the aged phenotype of the tissue (13).

Neurodegenerative disorders, such as Alzheimer's disease (AD) and Parkinson's disease (PD), are characterized by the progressive loss of neurons that ultimately leads to the decline of brain function, and the deterioration of cognition and/or locomotor activity $(14,15)$. Among the main risk factors of such disorders is the accumulation of protein aggregates, such as $\alpha$-synuclein, amyloid- $\beta$ and pathological tau proteins, as well as oxidative stress and mitochondrial dysfunction (16-18). Epigenetic modifications have been suggested to regulate neuroinflammation and subsequent neurodegeneration. Thus, several epigenetic biomarkers have emerged in brain diseases, such as patterns of methylation and the expression of specific microRNAs (miRNAs/miRs), as well as the levels of serum extracellular vesicle-derived circular RNAs $(19,20)$. The epigenome is dynamically regulated through diet and lifestyle, thus opening a novel avenue for neurodegenerative disorder therapeutics through the combination of pharmaceutical and nutritional strategies $(21,22)$.

Of note, a short telomere length has been shown to be associated with poor cognitive function and neurodegenerative diseases. Although the etiopathogenesis of neurodegenerative diseases has yet to be fully elucidated, telomere attrition and telomerase expression have emerged as novel mechanisms and potential therapeutic targets (19). Several telomerase activators exist, including both natural and synthetic compounds, acting through genetic manipulation mediated by adeno-associated viruses. A widely known natural compound is TA- $65^{\circledR}$ containing Astragalus membranaceus extract that has been shown to activate telomerase and to be associated with longer telomeres of peripheral blood monocytes and improved health parameters (20). Further analysis has demonstrated that cycloastragenol is the active compound responsible for the increased telomerase activity. Other known telomerase activators include product $\mathrm{B}$, a combination of antioxidants, and the synthetic GRN510, which has a similar structure to cycloastragenol (21). A previous comparative in vitro analysis of natural compounds identified a novel telomerase activator 08AGTL formulation containing Centella asiatica extract; this formulation was found to induce telomerase activation by up to 8.8 -fold compared with 2.2-fold in cells treated with TA-65 ${ }^{\circledR}(22)$. The present study aimed to investigate the potency of the novel natural formulation 'Reverse ${ }^{\mathrm{TM}}$ ' containing Centella asiatica extract, vitamin C, zinc and vitamin D3 on telomerase expression and telomerase activity in the brains of rats.

\section{Materials and methods}

Animal study design. Male Sprague-Dawley rats $(n=6$; 3 months old, with a body weight between 320 and $380 \mathrm{~g}$ ) and 18 male Sprague-Dawley rats (18 months old, with a body weight between 500 and $580 \mathrm{~g}$ ) obtained from the Animal Facility of the University of Medicine and Pharmacy of Craiova (Craiova, Romania) were included in the present study. The animal experiments were performed according to the EU Directive 2010/63/E.U. as amended by Regulation E.U.2019/1010 regarding animal experiments and approved by the Ethics Committee of the University of Medicine and Pharmacy of Craiova, Craiova Romania with the license no. 102/23.09.2019. Prior to the start of the experiment, the animals were allowed to acclimatize to their environment for 2 weeks. The animals were housed 2-3 animals per cage, with a 12-h dark/light cycle, and a constant humidity $(50 \pm 5 \%)$ and temperature $\left(21 \pm 2^{\circ} \mathrm{C}\right)$. During the study, the animals had free access to standard animal diet and tap water.

The animals were divided into four groups, with 6 animals per group, and received the treatment once per day via gavage for a period of 3 months, as presented in Table I. Based on power analysis, the minimum number of animals per group was estimated at 5; however, due to the fact that the experiments included middle-aged animals, 6 animals per group were used to prevent the unexpected loss of animals. The workflow of the study is presented in Fig. 1.

Treatment and dose selection. The treatment consisted of the administration of the 'Reverse ${ }^{\mathrm{TM}}$ ' supplement (Natural Doctor S.A), notified as a food supplement (Notification no. 6704/21-1-2020) at the Greek National Organization for Medicines under current legislation for food supplements.

The details regarding the dose selection have been previously described (10). Briefly, the animals from the treatment groups received 1 or 2 capsules/kg body weight/day of the 'Reverse ${ }^{\mathrm{TM}}$ ' supplement suspended in corn oil at a final volume of $1.5 \mathrm{ml}$. Each capsule contains $9 \mathrm{mg}$ Centella asiatica (L.) extract [consisting of a $>90 \%$ high purity single chemical entity, as assessed by high-performance liquid chromatography and gas chromatography (data not shown)], vitamin $\mathrm{C}$ (200 $\mathrm{mg}$ as magnesium ascorbate), zinc (5 mg as zinc citrate) and vitamin D3 (50 $\mu \mathrm{g}$ as cholecalciferol) per capsule.

The administered doses and treatment duration were in accordance with clinical recommendations relative 
Table I. Groups and corresponding treatments in the present study.

Animal groups $\quad$ Young control, $(n=6) \quad$ Old-aged control, $(n=6) \quad$ Old-aged group 1, $(n=6) \quad$ Old-aged group 2, $(n=6)$

Age of the animals

at the beginning

of the study (months)

Treatments
3

Corn oil
18

Corn oil
18

1 capsule/kg body weight of 'Reverse ${ }^{\mathrm{TM}}$,

(Natural Doctor S.A)
2 capsules/kg body weight of 'Reverse ${ }^{\mathrm{TM}}$, (Natural Doctor S.A)

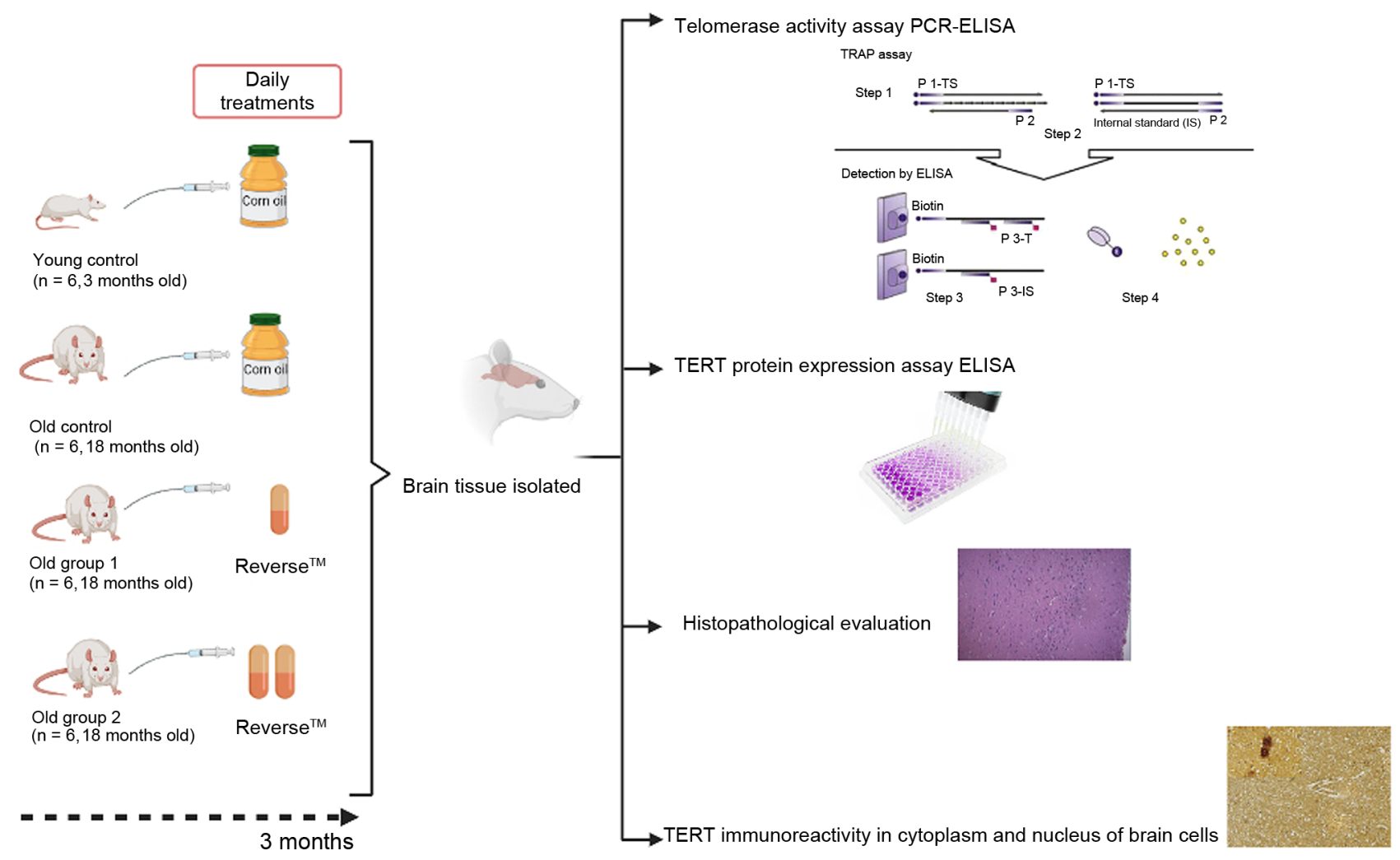

Figure 1. Workflow of the study design. TRAP, telomeric repeat amplification protocol; TERT, telomerase reverse transcriptase.

to body weight and with respect to previous studies for vitamin C $(23,24)$, vitamin D3 $(25,26)$ and zinc $(27)$. For Centella asiatica, no established clinical recommendations exist and the administered dose in the present study was markedly lower than that used in previous research (28).

The doses used for the rats were converted from those used for humans using the factor method. The allometric scaling approach, following the Food and Drug Administration guidelines, was applied for calculations and more specifically, the correction factor $(\mathrm{km})$ for rats used was equal to 6.2 and the safety factor value for converting rat doses to humans was equal to 10 (29). The dose values, as previously described (10) were calculated as follows: Rat dose $=$ human dose $\mathrm{x} 6.2 \times 10$, where human dose $=1 / 60$ capsule $/ \mathrm{kg} /$ body weight (reference human body weight $=60 \mathrm{~kg}(29)$.

For treatment group 1 corresponding to the human dose of 1 capsule $/$ day, the rat dose $=1 / 60 \times 6.2 \times 10=1.03$ capsule $/$ $\mathrm{kg} /$ bodyweight,approximate to 1 capsule $/ \mathrm{kg} /$ bodyweight perrat.
For treatment group 2, the rat dose $=2 / 60 \times 6.2 \times 10=2.06$ capsule $/$ $\mathrm{kg} /$ body weight, approximate to 2 capsules $\mathrm{kg} /$ body weight per rat.

The animals from the young and old-aged control group received only corn oil used as a solvent in a volume of $1.5 \mathrm{ml}$ once per day. The duration of the administration was set as 3 months that correspond to subchronic administration for rats. This was set based on a previous study by the authors using healthy human volunteers and using other vitamin supplements, where it was demonstrated that the modulation of telomerase and telomere length is usually observed following 6-12 months of treatment (7).

Brain collection. Following 3 months of treatment, the animals were sacrificed by exsanguination from the abdominal aorta under 5\% sevoflurane anesthesia. The brains were collected, and half were formalin-fixed for evaluation using immunohistochemistry and histopathology, and the other half of the 
cortex and cerebellum were separated and shock-frozen in small sections in liquid nitrogen and kept $-80^{\circ} \mathrm{C}$ until further analysis.

Telomerase activity assay. Telomerase activity in the cortex and cerebellum was measured using a commercial rat telomerase PCR-ELISA (TeloTAGGG Telomerase PCR ELISA PLUS; cat. no. 12013789 001, Roche Diagnostics), based on a photometric enzyme immunoassay for quantitative determination utilizing a telomeric repeat amplification protocol (TRAP) (30). TRAP reaction was performed according to the manufacturer's protocol using the Applied Biosystems PCR system (30 cycles at $50^{\circ} \mathrm{C}$; cat. no. 4485701 ; Applied Biosystems; Thermo Fisher Scientifiic, Inc.); the reactions were performed in triplicate. The detection of amplicons was further quantified using ELISA (StatFax 4700 ELISA reader system, Awareness Technology, Inc.). The absorbance values are reported as the $\mathrm{A}_{450 \mathrm{~nm}}$ reading against the blank. The mean of the absorbance reading of the negative control from those of the sample was extracted. Samples are regarded as telomerase-positive if the difference in the absorbance was $>0.2$ units.

TERT protein expression assay. The TERT levels in the cortex and cerebellum were measured using a commercial rat TERT sandwich ELISA kit (cat. no. SEC241Ra; Cloud-Clone Corp.) according to the manufacturer's instructions. Brain tissue homogenate was prepared as follows: After microdissection, the brain tissues (cortex and cerebellum) were weighted and homogenized in $0.5 \mathrm{ml}$ of $0.01 \mathrm{M}$ PBS (pH 7.0) using a tissue homogenizer on ice. The suspensions were subjected to two freeze/thaw cycles to further break the cell membranes and were centrifuged for $5 \mathrm{~min}$ at $500 \mathrm{xg}$ at $4^{\circ} \mathrm{C}$. The supernatant was stored at $-20^{\circ} \mathrm{C}$ until analysis (no more than 1 month).

TERT assay was performed using $100 \mu \mathrm{l}$ supernatant, according to the manufacturer's instructions and measured spectrophotometrically at a wavelength of $450 \mathrm{~nm}$ using an ELISA reader system (StatFax 4700, Awareness Technology, Inc.). The samples were examined in triplicate. The concentration of TERT expression in the sample was then determined by comparing the absorbance of the sample to the standard curve and normalized according to the protein concentration measured using Bradford reagent (Sigma-Aldrich).

Determination of TERT expression in brain tissue using immunohistochemistry. To analyze TERT expression in brain tissue, formalin-fixed, paraffin-embedded brain tissue was used. Brain tissue sections (10- $\mu \mathrm{M}$-thick) were deparaffinized and rehydrated in successive steps with a decreasing concentration of methanol. Antigen retrieval was performed using $1 \mathrm{mM}$ citrate buffer by microwaving for $10 \mathrm{~min}$ at $95^{\circ} \mathrm{C}$. Immunohistochemistry was performed as follows: i) The sections were blocked in PBS containing $10 \%$ normal goat serum at room temperature for $1 \mathrm{~h}$; ii) the sections were incubated overnight at $4^{\circ} \mathrm{C}$ with primary polyclonal rabbit anti-TERT antibody (diluted 1:100; cat. no. BS0233R, Bioss Antibodies); iii) appropriate universal immune-peroxidase polymer, anti-rabbit was applied (Histofine ${ }^{\circledR}$ Simple Stain Max PO anti-rabbit; diluted 1:10; cat. no. 414141F, Nichirei Bioscience) for $30 \mathrm{~min}$ at room temperature. Following staining with 3,3'-diaminobenzidine (DAB; Vector Laboratories, Inc.), the sections were dried overnight at $37^{\circ} \mathrm{C}$ and covered using mounting medium (Vector Laboratories, Inc.). The visualization and image collection of the slides was performed using a Nikon 90i motorized microscope equipped with a DS-Ri2 16 MP complementary metal oxide semiconductor (CMOS) camera and the Nikon NIS Elements AR software package Ver5.11.03 (Nikon Corporation).

Histopathological evaluation. Brain tissue was collected and kept for $48 \mathrm{~h}$ in a $4 \%$ paraformaldehyde solution. The samples were successively dehydrated in solution with increasing concentrations of ethanol $(1 \mathrm{~h} \mathrm{in} 70 \%$ solution, $1 \mathrm{~h}$ in $90 \%$ solution and $5 \mathrm{~h}$ in $100 \%$ solution) and then cleared in xylene for $2 \mathrm{~h}$. The tissue was then embedded in paraffin. From the paraffin block, sections of $25 \mu \mathrm{m}$ thickness were cut and stained with hematoxylin and eosin (Sigma-Aldrich) according to the standard protocol (31). The histopathological examination was performed under the Panthera $\mathrm{L}$ research microscope (Motic Europe, S.L.U.) with the licensed Panthera L photomicrographic image acquisition software Motic Images Plus 2.0ML for Microsoft Windows Legacy Version w/o MI Devices (Motic Europe, S.L.U.).

Statistical analysis. Statistical analysis was performed using GraphPad Prism version 5.0 software for Windows (GraphPad Software, Inc.). One-way analysis of variance (ANOVA; parametric) for independent samples was used for the comparisons of the mean values for more than two independent groups. One-way ANOVA was followed by post hoc analysis using the Bonferroni adjusted $t$-test. A P-value $\leq 0.05$ was considered to indicate a statistically significant difference.

\section{Results}

Telomerase activity. The telomerase activity levels were significantly lower in the brain cortex of the old-aged control group $(0.17 \pm 0.01 ; \mathrm{P}<0.05)$ compared with the young control group $(0.25 \pm 0.01 ; \mathrm{P}<0.05)$ at 3 months following the initiation of the study. Similar results were revealed by the analysis of the cerebellum where the telomerase activity levels were significantly lower in the old-aged control group (21 months old) compared with the young control group $(0.15 \pm 0.02$ vs. $0.26 \pm 0.02 ; \mathrm{P}<0.05)$ at 3 months following the initiation of the study. The telomerase activity levels were significantly increased in the old-aged groups that received the 'Reverse ${ }^{\mathrm{TM}}$, supplement compared with the untreated old-aged control group, both in the cortex and cerebellum $(\mathrm{P}<0.05)$. No significant differences were observed in the telomerase activity between the old-aged groups 1 and 2, and the young control group (Fig. 2).

TERT protein level. The TERT protein levels were significantly lower in the old-aged control group compared with the young control group in both the cortex and cerebellum ( $\mathrm{P}<0.05$; Fig. 3). Treatment with 2 capsules/kg body weight of the 'Reverse ${ }^{\mathrm{TM}}$ ' supplement for 3 months resulted in a significant increase in TERT protein levels in the old-aged group 2 compared with the old-aged control group in both the cortex and cerebellum $(\mathrm{P}<0.05$; Fig. 3$)$. No statistically significant 


\section{A Brain tissue (cerebellum)}

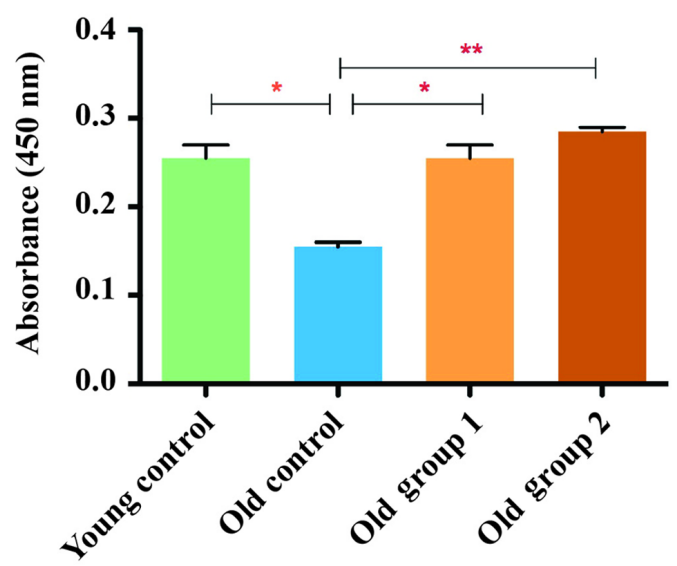

\section{B Brain tissue (cortex)}

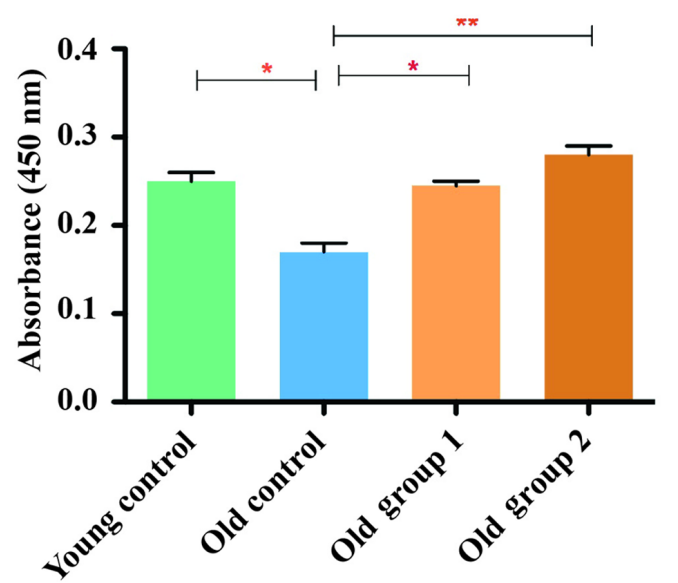

Figure 2. Telomerase activity following 3 months of treatment expressed as absorbance units (A450 nm). (A) In the cerebellum and (B) in the cortex. ${ }^{*} \mathrm{P}<0.05$ and ${ }^{* *} \mathrm{P}<0.01$.

\section{A Brain tissue (cerebellum)}

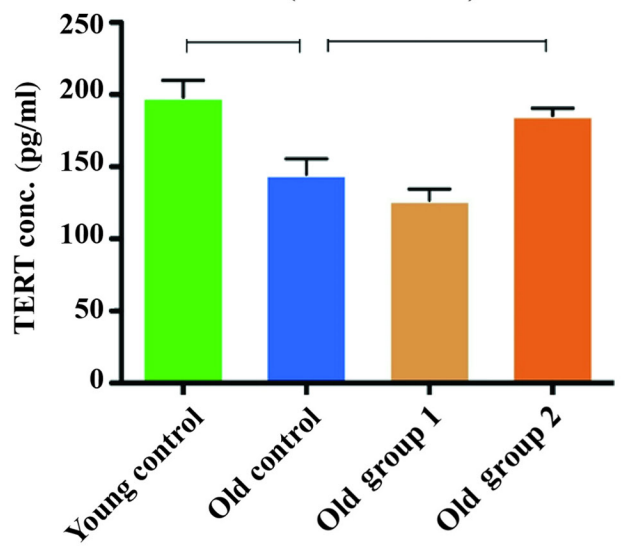

\section{B Brain tissue (cortex)}

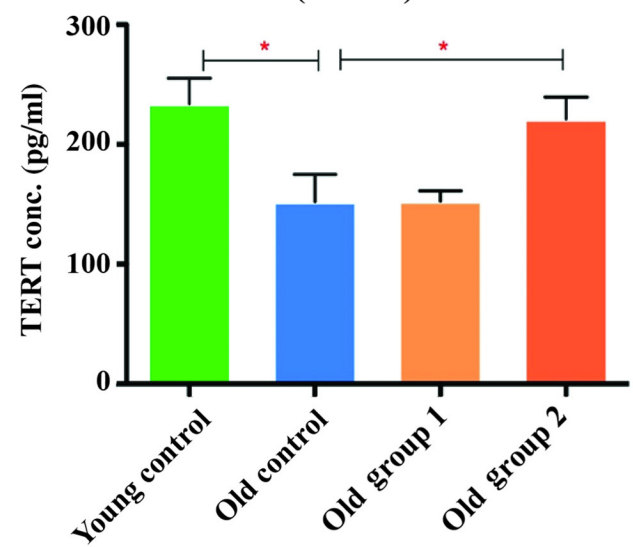

Figure 3. TERT protein level in (A) the cerebellum; and (B) the cortex. ${ }^{*} \mathrm{P}<0.05$. TERT, telomerase reverse transcriptase.

A

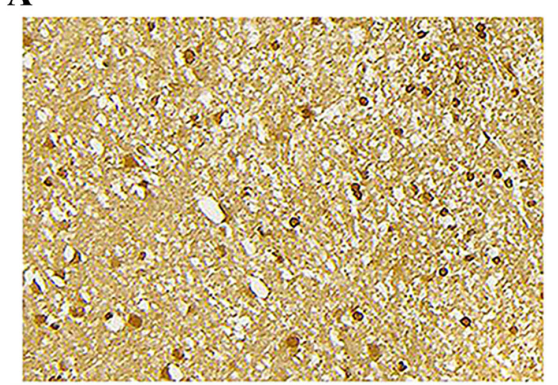

C

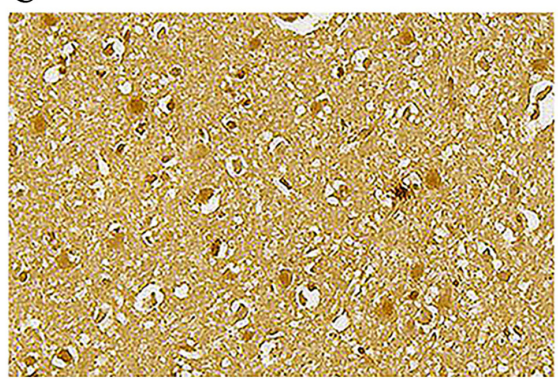

B

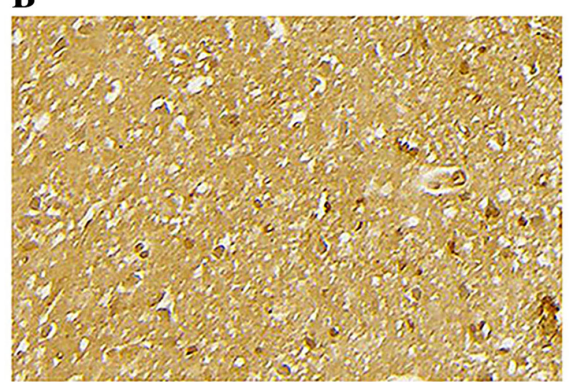

D

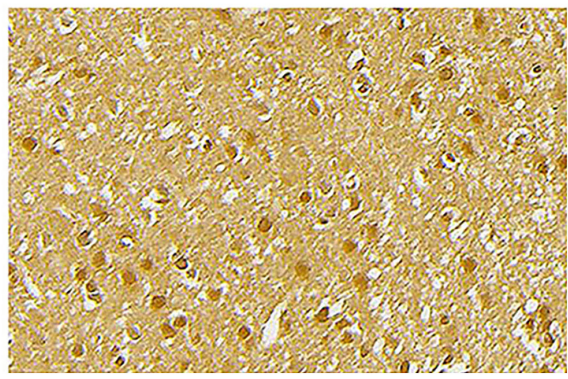

Figure 4. Telomerase reverse transcriptase immunoreactivity in the cytoplasm and nucleus of brain cells in the cortex (brown color; magnification, $\mathrm{x} 20$ ), 3,3'-diaminobenzidine staining. (A) Young control group; (B) old-aged control group; (C) old-aged group 1; (D) old-aged group 2. 
A

B

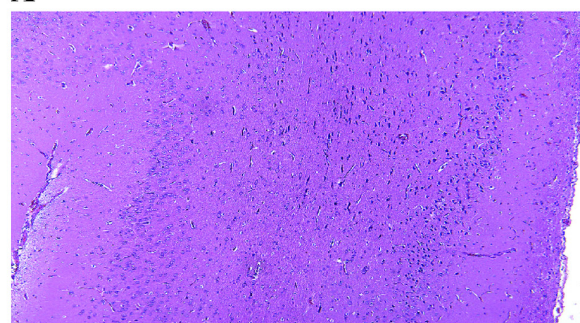

C

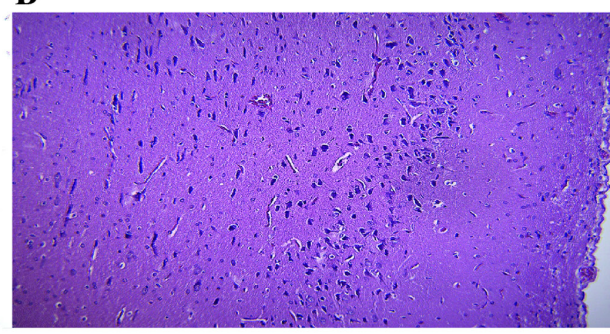

D

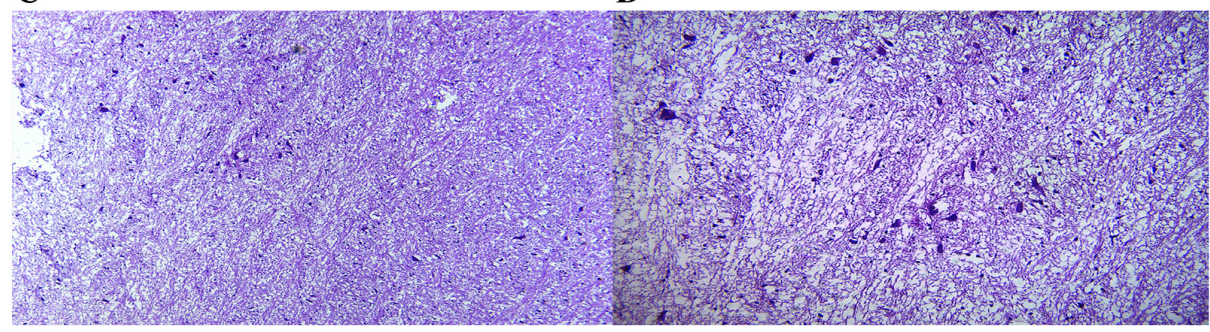

Figure 5. Microscopic structure of the cerebral cortex. (A) Young control group. Fragment with the microscopic structure of the cerebral cortex with normal differentiation. H\&E staining; magnification, x100. (B) Young control group. More detailed view of the image in panel A. H\&E staining; magnification, $\mathrm{x} 200$. (C) Old-aged control group. Fragment with the microscopic structure of the cerebral cortex with cytoarchitectonic structure modified by edema and a poor neuronal cell distribution is shown. H\&E staining; magnification, x100. (D) Old-aged control group. More detailed view of the image in panel C, with rare large and medium pyramidal neurons dissociated by marked edema. H\&E staining; magnification, x200. H\&E, hematoxylin and eosin.

A

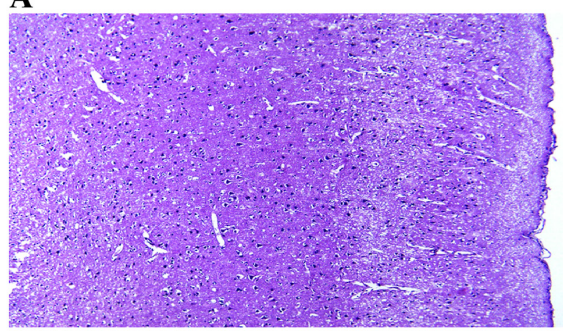

C
B

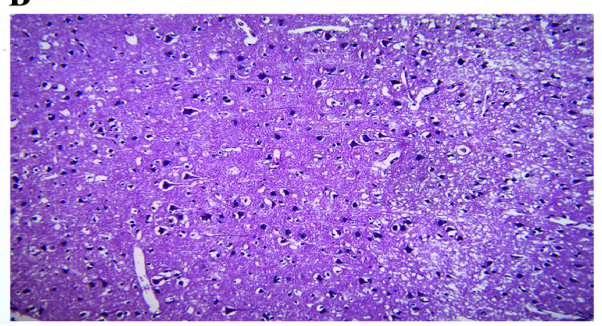

D

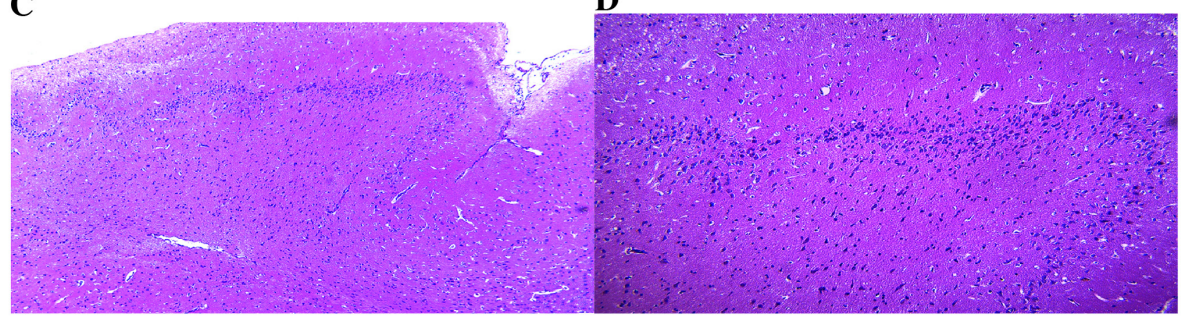

Figure 6. Microscopic structure of the cerebral cortex. (A) Old-aged group 1. Fragment of the microscopic structure of cerebral cortex with cytoarchitectonic structure modified by edema and poor neuronal cellular distribution is shown. H\&E staining; magnification, x100. (B) Old-aged group 1. More detailed view of the image in panel A, with the identification of cerebral cortex layers. H\&E staining; magnification, x200. (C) Old-aged group 2. Fragment of the microscopic structure of the cerebral cortex with relative normal cytoarchitectonic structure is shown. H\&E staining; magnification, $\mathrm{x} 100$; (D) More detailed view of the image in panel $\mathrm{C}$, with the identification of the deep layers of the cerebral cortex. H\&E staining; magnification, $\mathrm{x} 200 . \mathrm{H} \& \mathrm{E}$, hematoxylin and eosin.

differences in TERT protein levels were observed between the old-aged control group and old-aged group 1 in both the cortex and cerebellum tissue (Fig. 3). Staining with anti-TERT antibody revealed that TERT immunoreactivity was present in the cytoplasm and nucleus of the brain cells in the rat cortex stained with DAB (Fig. 4).

\section{Histopathological evaluation}

Histopathological aspect of the cerebral cortex. The microscopic evaluation of the rat cerebral cortex of the old-aged control group, revealed an increase in edema that dissociated the layers of the cerebral cortex and appeared as an unequal distribution with cytoarchitectonics modified by poor neuronal cellularity. Several different areas of each cerebral cortex were observed and the results were similar; thus, the most representative images are presented (Fig. 5C and D). By contrast, the young control group exhibited a normal microscopic structure (Fig. 5A and B). Treatment with 1 capsule/kg body weight of the 'Reverse ${ }^{\mathrm{TM}}$ ' supplement for 3 months led to a slight decrease in edema; however, the cytoarchitectonic structure remained altered and a poor neuronal cellular distribution was observed (Fig. 6A and B). In the old-aged group 2, following treatment with 2 capsules $/ \mathrm{kg}$ body weight of the 'Reverse ${ }^{\mathrm{TM}}$, supplement for 3 months, the cytoarchitecture of the cerebral 
A

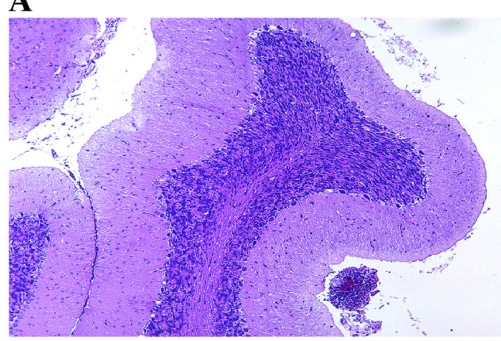

C

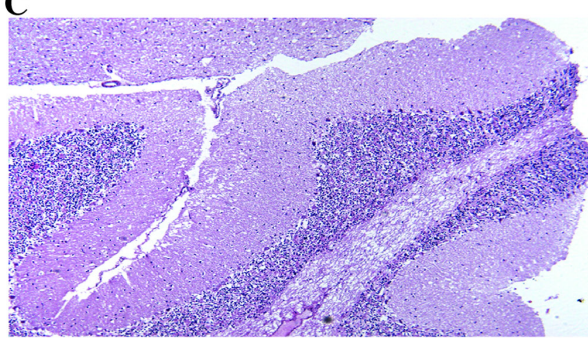

B

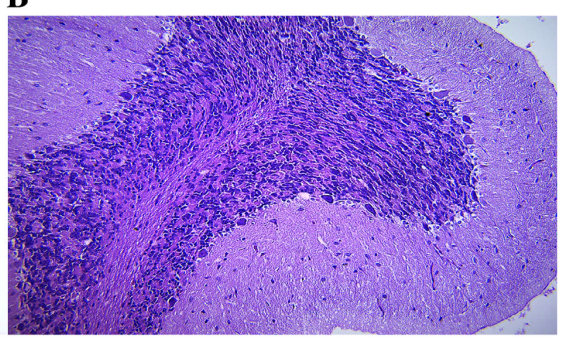

D

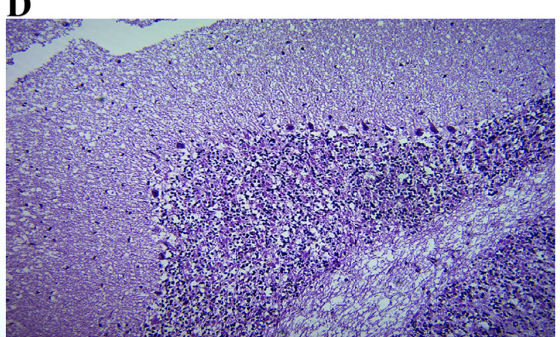

Figure 7. Microscopic structure of the cerebellar cortex. (A) Young control group. Fragment of the microscopic structure of cerebellar cortex with normal differentiation is shown. H\&E staining; magnification, x100. (B) Young control group. More detailed view of the image in panel A, in which the layers of the cerebellar cortex are observed .H\&E staining; magnification, x200. (C) Old-aged control group. Fragment of the microscopic structure of the cerebellar cortex with the modified architecture of the layers of the cerebellar cortex is shown. H\&E staining; magnification, x100; (D) Old-aged control group. More detailed view of the image in panel $\mathrm{C}$, in which the modification of the architecture of the layers of the cerebellar cortex is observed, with increased interneuronal edema. H\&E staining; magnification, x200. H\&E, hematoxylin and eosin.

A

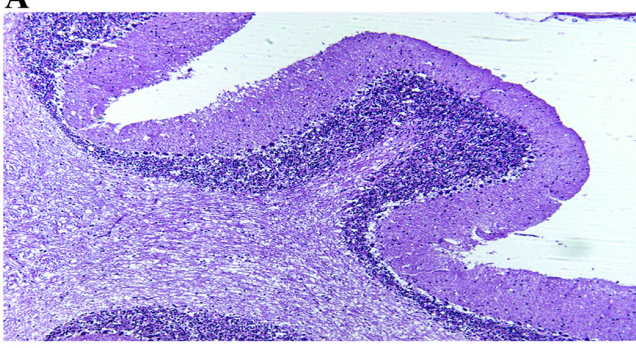

C

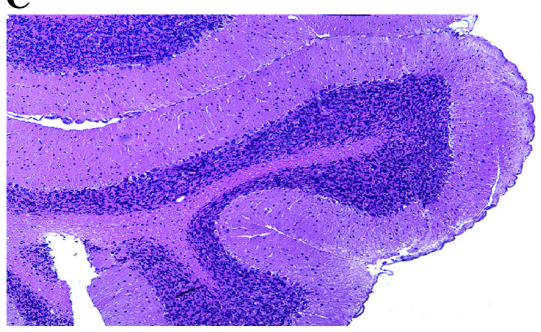

B

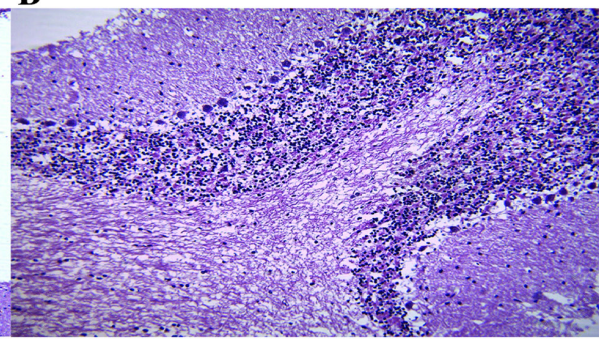

D

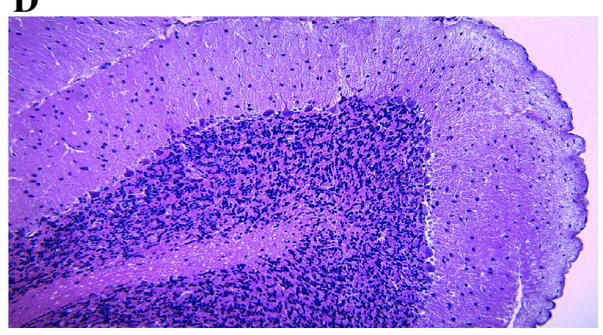

Figure 8. Microscopic structure of the cerebellar cortex. (A) Old-aged group 1. Fragment of the microscopic structure of the cerebellar cortex with the modified architecture of the layers of the cerebellar cortex with discrete interneuronal edema is shown. H\&E staining; magnification, x100. (B) Old-age group 1. More detailed view of the image in panel A. H\&E staining; magnification, x200. (C) Old-aged group 2. Fragment of the microscopic structure of cerebellar cortex with a relatively normal cytoarchitectonic structure is shown. H\&E staining; magnification, $\mathrm{x} 100$. (D) More detailed view of the image in panel C revealing the layers of the cerebellar cortex. H\&E staining; magnification, x200. H\&E, hematoxylin and eosin.

cortex appeared relatively normal, similar to the differentiation observed in the young control group and the edema was discrete in the intermediate layers of the cerebral cortex (Fig. 6C and D).

Microscopic aspect of the cerebellar cortex. The microscopic evaluation of the rat cerebellar cortex of the old-aged control group revealed an increase in edema that dissociated the layers of the cerebellar cortex; a modified cytoarchitecture was also observed that was accentuated at the level of the cerebellar layers, although not in the same measure as the one identified in the cerebral cortex. Several different areas of each cerebellar cortex were observed and the results were similar; thus, the most representative images are presented (Fig. 7C and D). By contrast, the young control group exhibited a normal microscopic structure (Fig. 7A and B). In the group treated with 1 capsule/kg body weight of the 'Reverse ${ }^{\mathrm{TM}}$, supplement for 3 months (old-aged group 1) the modified cytoarchitecture at the level of the cerebellar layers, through edema, remained and the effects were slightly milder compared with the those in the old-aged control group (Fig. 8A and B). In old-aged group 2 that received 2 capsules $/ \mathrm{kg}$ body weight of the 'Reverse ${ }^{\mathrm{TM}}$ ' supplement for 3 months, the cytoarchitecture 
of the cerebellar cortex was relatively normal, similar to the differentiation observed in the young control group, and the edema was discrete in the intermediate layers of the cerebellar cortex (Fig. 8C and D).

\section{Discussion}

The present study aimed to investigate the activity of telomerase and the expression of TERT in the brains of rats treated with a novel telomerase activator containing Centella asiatica extract, vitamin $\mathrm{C}$, zinc and vitamin D3 for 3 months.

Brain ageing is a key risk factor and mechanism responsible for a number of neurodegenerative diseases $(32,33)$, and it has been demonstrated that telomerase activity and TERT abundance may play a protective role in neurons and brain cell functions (34).

To the best of our knowledge, the present study demonstrates for the first time that the administration of the dietary supplement 'Reverse ${ }^{\mathrm{TM}}$ ' containing Centella asiatica extract, vitamin $\mathrm{C}$ (as magnesium ascorbate), zinc (as zinc citrate) and vitamin D3 (as cholecalciferol), to 18-month-old rats for 3 months, restores the decline in TERT expression and enhances telomerase activity. The findings presented herein demonstrated that the administration of the dietary supplement 'Reverse ${ }^{\mathrm{TM}}$ ' resulted in a moderate increase in TERT expression, and the telomerase activity was highly increased between old-aged groups 1 and 2, and the young control group. The findings also demonstrated that the activation of telomerase activity could be achieved despite the limited increase in TERT expression. However, further studies using larger-sized groups are warranted to establish whether there is a dose-response association. These findings are in line with those in a previous study by the authors which demonstrated that 'Reverse ${ }^{\mathrm{TM}}$ ' improved motor performance and decreased the stress levels of rats (10).

Specifically, in the present study, telomerase activity and TERT expression were shown to be significantly lower in 21-month-old rats compared with 6-month-old rats in both the brain cortex and cerebellum, suggesting the age-dependent expression of TERT in rats, in line with other findings (10). The TERT levels regulate telomerase activity, and it has been described that telomerase activity decreases during embryogenesis. By contrast, the TERT levels are maintained, at least in humans, possibly to protect neurons from oxidative stress and neurodegeneration (34).

The dose of 1 capsule of 'Reverse ${ }^{\mathrm{TM}}$ ' supplement per $\mathrm{kg}$ body weight per rat (old-aged group 1) resulted in a statistically significant increase in telomerase activity in the cortex and cerebellum of 21-month-old rats. Comparable results were obtained for TERT expression, although these did not reach statistical significance. The effect was more evident with the 2-capsule dose (old-aged group 2) where the levels of telomerase activity were significantly higher than those in the old-aged control group, reaching the levels of the young group both in the cortex and the cerebellum of the rats. Accordingly, the TERT expression levels were higher in the old-aged group 2 than in the old-aged group 1 and the old-aged control group. Anti-TERT immunohistochemistry staining in the cortex and cerebellum tissue slides confirmed the aforementioned results. These findings suggest that the oral administration of
2 capsules/kg body weight per rat of the 'Reverse ${ }^{\mathrm{TM}}$ ' dietary supplement is more effective for telomerase activation and TERT level restoration in the brain than the 1 capsule dose.

The same microscopic changes produced by aging were noted following the comparative analysis performed on the cerebral and cerebellar cortex. The edema was constant and accentuated in both types of cortex with a modified cytoarchitecture and poor neuronal cellularity in the 21-month-old rats. Previous research supports these findings. Desbordes and Cohadon (35) demonstrated that in rat brains, cellular swelling increased with aging, while extracellular edema was less evident. These findings can be explained by the decreased activity of $\mathrm{Na}^{+}-\mathrm{K}^{+}$-ATPase activity. It has been demonstrated that at 3 months of age, which corresponds to the end of adolescence, rats reach the peak number of neurons both in the cerebellum and cortex. From this age on, the progressive decline in the number of neurons commences (36). Morterá and Herculano-Houzel (37) demonstrated that by 22 months of age, the decrease in neurons in the cerebellum and cortex of rats is almost $30 \%$ less than the value observed at the age of 3 months. In the present study, the administration of 1 capsule $/ \mathrm{kg}$ body weight of the 'Reverse ${ }^{\mathrm{TM}}$ ' supplement for 3 months exerted only a slight beneficial effect on the decline in the number of neurons. The administration of 2 capsules $/ \mathrm{kg}$ body weight of the 'Reverse ${ }^{\mathrm{TM}}$ ' supplement for 3 months to the 18-month-old rats (old-aged group 2) exerted a structural reversibility effect close to the differentiation of the gray matter from the young control group that is explained by telomerase activation and the restoration of TERT levels in the brain observed in this group. It has been demonstrated that TERT inhibits the death of cells and promote the survival of neurons (38). Even if neurons are non-dividing cells, telomerase expression in neurons plays an additional role in regulating apoptosis beyond its function in telomere maintenance (39).

Previous studies on telomerase expression enhancement in the brain have been conducted either via genetic intervention or by the use of synthetic or natural enzyme activators, as reviewed by Saretzki and Wan (34). As regards natural activators, TA- $65^{\circledR}$ and GRN510 have yielded encouraging results on the beneficial effects of telomerase activation on brain function. Specifically, as previously demonstrated, the administration of TA- $65^{\circledR}$ to aged mice ameliorated several physiological parameters, including neuromuscular coordination and cognition accompanied by the increased expression of the mouse TERT (mTERT). Furthermore, treatment with TA- $65^{\circledR}$ and GRN510 yielded similar results in brain-specific TERT expression, but only TA- $65^{\circledR}$ decreased mitochondrial ROS accumulation and $\alpha$-synuclein aggregates (40). However, telomerase activation with TA- $65^{\circledR}$, which contains Astragalus membranaceus extract, has been shown to be significantly lower than with formulations including Centella asiatica extract (22). 'Reverse ${ }^{\mathrm{TM}}$ ', is a dietary supplement containing Centella asiatica extract, vitamin C, vitamin D3 and zinc, constituents with known antioxidant properties previously described in terms of their relevance to the present study (10). In particular, Centella asiatica has been shown to exert neuroprotective effects, while enhancing cognitive function (41). Vitamin $\mathrm{C}$ and zinc have been reported to play a neuroprotective role and function synergistically to improve synaptic activity and detoxification, and to maintain the physiological 
function of protein degradation mediated by ubiquitin (42). Vitamin D deficiency is a known and well-described risk factor for several neurodegenerative diseases $(43,44)$. Vitamin D has been described as a key neuroprotector through its role as an immunomodulator, a regulator of calcium levels in neurons and a mediator of the detoxification mechanism $(45,46)$. Telomerase activity has been reported to be enhanced in previous in vivo studies on vitamin $\mathrm{C}$, vitamin $\mathrm{D}$, zinc and Centella asiatica $(47,48)$. However, additional studies are required to determine whether the combination of these compounds acts synergistically in terms of telomerase activation and to investigate the underlying molecular mechanisms. Overall, the beneficial effects of natural products on oxidative stress and inflammation is a widely studied field; thus, they have emerged as potential therapeutic targets in several neurodegenerative disorders. Indeed, there is evidence to indicate that phytochemicals, such as polyphenols, found in pomegranate (47), aloe vera, red fruits and other natural products are important for normal brain function $(15,17,49-51)$. Detailed analyses of the different modes of action of natural phytochemicals in AD have suggested that certain compounds may serve as potent therapeutic agents as they are effective, easy-to access, safe and lower-cost options compared to other drugs $(14,32,52,53)$.

Although not aiming to extrapolate the present findings, it may be noted that the beneficial effects of 'Reverse ${ }^{\mathrm{TM}}$ ' on telomerase/TERT levels may be mediated by the reduction of oxidative stress in the brain microenvironment through the synergistic effects of the formulation constituents. However, a limitation of the present study is the lack of oxidative stress damage measurements and the comparative analysis of separate compounds contained in 'Reverse ${ }^{\mathrm{TM}}$ ' to validate this hypothesis. As regards the synergy of nutrients, there is significant evidence to indicate that combined treatment with nutraceuticals may prove to be extremely useful in the management of age-related diseases $(49,54,55)$. Specifically for brain health, it has been demonstrated that the administration of a multi-nutrient supplement can prevent brain ischemia more potently than single nutrients in vitro (56). Future studies are required however to validate the additive effects of 'Reverse ${ }^{\mathrm{TM}}$ ', constituents on TERT expression and telomerase activity. Further studies are also required to investigate the association between telomere length, telomerase activity and motor function post-treatment with 'Reverse ${ }^{\mathrm{TM}}$ ', to provide mechanistic insight into aging prevention and management strategies for common neurodegenerative diseases.

The clinical relevance of the present study refers to the potency of 'Reverse ${ }^{\mathrm{TM}}$ ' to increase TERT expression and telomerase activity in the brains of middle-aged rats. Middle-aged rats at 18 months old correspond to 45-year-old humans; thus, rats at this age represent a good experimental model to examine the effects of the intervention on the early-aged brain that could be used as a tool to delay neurodegenerative diseases (57). The results of the present study demonstrate that the increase inh TERT expression in the brains of middle-aged rats and the increase in telomerase activity can be achieved through nutraceutical supplementation, and not only in the postnatal or embryonic period [when central nervous system (CNS) development occurs]. The reactivation of such pathways that are active during CNS development can improve functional outcomes following brain lesions, such as stroke or neurodegenerative diseases, and may prove to play a crucial role in the management of a number of CNS diseases.

$\mathrm{AD}$ and PD are slowly progressive neurodegenerative disorders that occur upon irreversible brain damage, extensive inflammation and oxidative stress, leading to dementia and motor/non-motor traits, respectively. In AD, telomere attrition emerges as a critical mechanism possibly related to the increased oxidative stress that precedes the accumulation of amyloid and neurofibrillary tangles (58). TERT expression inversely correlates with tau expression in neurons, indicating an oxidative stress-mediated mechanism of AD. AD can occur during the later or early stages of life (known as early-onset AD) and occurs prior to the age of 60 . There is evidence to indicate that midlife interventions focusing on modifiable risk factors can prevent dementia (59). Accordingly, PD etiopathogenesis includes the accumulation of $\alpha$-synuclein caused by folding impairment and its defective clearance through the autophagic pathway. It has been demonstrated that telomerase activators can successfully facilitate the normal function of autophagy and the reduction of protein aggregates, possibly mediated by the increase in TERT levels. In addition, TERT is known for its non-canonical function as an antioxidant defense mediator, which further supports the physiological protein post-translational folding and mitochondrial function (60).

In conclusion, to the best of our knowledge, the present study provides the first evidence of the potency of a novel telomerase activator based on the formulation 'Reverse ${ }^{\mathrm{TM}}$, containing Centella asiatica extract, vitamin C, vitamin D3 and zinc as a treatment against brain aging and related disorders using an in vivo animal model. These results may have a positive impact on the management of a number of CNS diseases. However, they need to be further validated in clinical trials to establish the dosing and duration in order to achieve the optimal therapeutic effects.

\section{Acknowledgements}

Not applicable.

\section{Funding}

No funding was received.

\section{Availability of data and materials}

The datasets used and/or analyzed during the current study are available from the corresponding author on reasonable request.

\section{Authors' contributions}

DC and DT designed the study and wrote the manuscript as a special part of a $\mathrm{PhD}$ thesis from the University of Medicine and Pharmacy of Craiova, Romania. AMB, AOD, ES, RM, ER, LC, DAS, IR and MN performed the analyses, statistical analysis and presentation of the results and wrote the manuscript. DT, AT, DAS, IR, AMB, MN and DC critically reviewed the text and prepared the figures. AT, AOD, AMB and DC critically assessed the design of the study and the interpretation of the findings. DT, AMB, AOD confirm the authenticity of 
all the raw data. All authors have read and approved the final version of the manuscript.

\section{Ethics approval and consent to participate}

The present study was conducted according to the guidelines of the Declaration of Helsinki, and approved by the Ethical Committee of the University of Medicine and Pharmacy of Craiova, Craiova, Romania (no. 102/23.09.2019). All the procedures used in this experiment were according to the European directives for the animal experiments (EU Directive 2010/63/EU as amended by Regulation EU 2019/1010).

\section{Patient consent for publication}

Not applicable.

\section{Competing interests}

DAS is the Editor-in-Chief for the journal, but had no personal involvement in the reviewing process, or any influence in terms of adjudicating on the final decision, for this article. DT is a scientific advisor for Natural Doctor S.A. The other authors declare that they have no competing interests.

\section{References}

1. de Lange T: T-loops and the origin of telomeres. Nat Rev Mol Cell Biol 5: 323-329, 2004

2. Miwa S, Czapiewski R, Wan T, Bell A, Hill KN, von Zglinicki T and Saretzki G: Decreased mTOR signalling reduces mitochondrial ROS in brain via accumulation of the telomerase protein TERT within mitochondria. Aging (Albany NY) 8 : 2551-2567, 2016

3. Singhapol C, Pal D, Czapiewski R, Porika M, Nelson G and Saretzki GC: Mitochondrial telomerase protects cancer cells from nuclear DNA damage and apoptosis. PLoS One 8: e52989, 2013.

4. Martens A, Schmid B, Akintola O and Saretzki G: Telomerase Does Not Improve DNA Repair in Mitochondria upon Stress but Increases MnSOD Protein under Serum-Free Conditions. Int J Mol Sci 21: 21, 2019.

5. Rosen J, Jakobs P, Ale-Agha N, Altschmied J and Haendeler J: Non-canonical functions of Telomerase Reverse Transcriptase - Impact on redox homeostasis. Redox Biol 34: 101543-101543, 2020.

6. Padureanu R, Albu CV, Mititelu RR, Bacanoiu MV, Docea AO, Calina D, Padureanu V, Olaru G, Sandu RE, Malin RD, et al: Oxidative Stress and Inflammation Interdependence in Multiple Sclerosis. J Clin Med 8: 11, 2019.

7. Tsoukalas D, Fragkiadaki P, Docea AO, Alegakis AK, Sarandi E, Vakonaki E, Salataj E, Kouvidi E, Nikitovic D, Kovatsi L, et al: Association of nutraceutical supplements with longer telomere length. Int J Mol Med 44: 218-226, 2019.

8. Tsoukalas D, Fragoulakis V, Sarandi E, Docea AO, Papakonstaninou E, Tsilimidos G, Anamaterou C, Fragkiadaki P, Aschner M, Tsatsakis A, et al: Targeted Metabolomic Analysis of Serum Fatty Acids for the Prediction of Autoimmune Diseases. Front Mol Biosci 6: 120, 2019.

9. Tsoukalas D, Sarandi E, Thanasoula M, Docea AO, Tsilimidos G, Calina D and Tsatsakis A: Metabolic Fingerprint of Chronic Obstructive Lung Diseases: A New Diagnostic Perspective. Metabolites 9: 18, 2019.

10. Tsoukalas D, Zlatian O, Mitroi M, Renieri E, Tsatsakis A, Izotov BN, Burada F, Sosoi S, Burada E, Buga AM, et al: A Novel Nutraceutical Formulation Can Improve Motor Activity and Decrease the Stress Level in a Murine Model of Middle-Age Animals. J Clin Med 10: 624, 2021.

11. Palm $\mathrm{W}$ and de Lange $\mathrm{T}$ : How shelterin protects mammalian telomeres. Annu Rev Genet 42: 301-334, 2008.
12. Victorelli S and Passos JF: Telomeres and Cell Senescence - Size Matters Not. EBioMedicine 21: 14-20, 2017.

13. Carneiro MC, de Castro IP and Ferreira MG: Telomeres in aging and disease: Lessons from zebrafish. Dis Model Mech 9: 737-748, 2016.

14. Calina D, Buga AM, Mitroi M, Buha A, Caruntu C, Scheau C, Bouyahya A, El Omari N, El Menyiy N and Docea AO: The Treatment of Cognitive, Behavioural and Motor Impairments from Brain Injury and Neurodegenerative Diseases through Cannabinoid System Modulation-Evidence from In Vivo Studies. J Clin Med 9: 28, 2020.

15. Salehi B, Sestito S, Rapposelli S, Peron G, Calina D, Sharifi-Rad M, Sharopov F, Martins N and Sharifi-Rad J: Epibatidine: A Promising Natural Alkaloid in Health. Biomolecules 9: 10, 2018.

16. Salehi B, Quispe C, Chamkhi I, El Omari N, Balahbib A, Sharifi-Rad J, Bouyahya A, Akram M, Iqbal M, Docea AO, et al: Pharmacological Properties of Chalcones: A Review of Preclinical Including Molecular Mechanisms and Clinical Evidence. Front Pharmacol 11: 592654, 2021.

17. Salehi B, Sharifi-Rad J, Cappellini F, Reiner Ž, Zorzan D, Imran M, Sener B, Kilic M, El-Shazly M, Fahmy NM, et al: The Therapeutic Potential of Anthocyanins: Current Approaches Based on Their Molecular Mechanism of Action. Front Pharmacol 11: 1300, 2020.

18. Radu G, Bordejevic AD, Buda V, Tomescu MC, Dragan I, Dehelean L, Cocos IL, Cheveresan A and Andor M: Cardiovascular risk factors for different types of psychiatric pathologies. A correlative study. Farmacia 68: 835-842, 2020.

19. Lex K, Maia Gil M, Lopes-Bastos B, Figueira M, Marzullo M, Giannetti K, Carvalho T and Ferreira MG: Telomere shortening produces an inflammatory environment that increases tumor incidence in zebrafish. Proc Natl Acad Sci USA 117: 15066-15074, 2020.

20. Liu P, Zhao H and Luo Y: Anti-Aging Implications of Astragalus membranaceus (Huangqi): A Well-Known Chinese Tonic. Aging Dis 8: 868-886, 2017.

21. Harley CB, Liu W, Flom PL and Raffaele JM: A natural product telomerase activator as part of a health maintenance program: Metabolic and cardiovascular response. Rejuvenation Res 16: 386-395, 2013.

22. Tsoukalas D, Fragkiadaki P, Docea AO, Alegakis AK, Sarandi E, Thanasoula M, Spandidos DA, Tsatsakis A, Razgonova MP and Calina D: Discovery of potent telomerase activators: Unfolding new therapeutic and anti-aging perspectives. Mol Med Rep 20: 3701-3708, 2019

23. Institute of Medicine (US) Panel on Dietary Antioxidants and Related Compounds: Dietary Reference Intakes for Vitamin C, Vitamin E, Selenium, and Carotenoids. National Academies Press (US), Washington, DC, 2000.

24. Sil S, Ghosh T, Gupta P, Ghosh R, Kabir SN and Roy A: Dual Role of Vitamin C on the Neuroinflammation Mediated Neurodegeneration and Memory Impairments in Colchicine Induced Rat Model of Alzheimer Disease. J Mol Neurosci 60: 421-435, 2016.

25. Holick MF, Binkley NC, Bischoff-Ferrari HA, Gordon CM, Hanley DA, Heaney RP, Murad MH and Weaver CM: Guidelines for preventing and treating vitamin D deficiency and insufficiency revisited. J Clin Endocrinol Metab 97: 1153-1158, 2012.

26. Williamson L, Hayes A, Hanson ED, Pivonka P, Sims NA and Gooi JH: High dose dietary vitamin D3 increases bone mass and strength in mice. Bone Rep 6: 44-50, 2017.

27. Institute of Medicine (US) Panel on Micronutrients: Dietary Reference Intakes for Vitamin A, Vitamin K, Arsenic, Boron, Chromium, Copper, Iodine, Iron, Manganese, Molybdenum, Nickel, Silicon, Vanadium, and Zinc. National Academies Press (US), Washington, DC, 2001.

28. Rao SB, Chetana M and Uma Devi P: Centella asiatica treatment during postnatal period enhances learning and memory in mice. Physiol Behav 86: 449-457, 2005.

29. Nair AB and Jacob S: A simple practice guide for dose conversion between animals and human. J Basic Clin Pharm 7: 27-31, 2016.

30. Grin Y, Admoni T and Priel E: Telomerase activity in the various regions of mouse brain: Non-radioactive telomerase repeat amplification protocol (TRAP) assay. J Vis Exp 91: e51865, 2014.

31. Iordache AM, Buga AM, Albulescu D, Vasile RC, Mitrut R, Georgiadis G, Zisis IE, Mamoulakis C, Tsatsakis A, Docea AO, et al: Phosphodiesterase-5 inhibitors ameliorate structural kidney damage in a rat model of contrast-induced nephropathy. Food Chem Toxicol 143: 111535, 2020. 
32. Salehi B, Prakash Mishra A, Nigam M, Karazhan N, Shukla I, Kiełtyka-Dadasiewicz A, Sawicka B, Głowacka A, Abu-Darwish MS, Hussein Tarawneh A, et al: Ficus plants: State of the art from a phytochemical, pharmacological, and toxicological perspective. Phytother Res 35: 1187-1217, 2021.

33. Aloizou AM, Siokas V, Pateraki G, Liampas I, Bakirtzis C, Tsouris Z, Lazopoulos G, Calina D, Docea AO, Tsatsakis A, et al: Thinking outside the Ischemia Box: Advancements in the Use of Multiple Sclerosis Drugs in Ischemic Stroke. J Clin Med 10: 19, 2021.

34. Saretzki G and Wan T: Telomerase in Brain: The New Kid on the Block and Its Role in Neurodegenerative Diseases. Biomedicines 9: 490, 2021.

35. Desbordes P and Cohadon F: Brain water and aging. J Gerontol 42 : 655-659, 1987.

36. Spear LP: The adolescent brain and age-related behavioral manifestations. Neurosci Biobehav Rev 24: 417-463, 2000.

37. Morterá P and Herculano-Houzel S: Age-related neuronal loss in the rat brain starts at the end of adolescence. Front Neuroanat 6 : 45,2012 .

38. Saretzki G: Telomerase, mitochondria and oxidative stress. Exp Gerontol 44: 485-492, 2009.

39. Ahmed S, Passos JF, Birket MJ, Beckmann T, Brings S, Peters H, Birch-Machin MA, von Zglinicki T and Saretzki G: Telomerase does not counteract telomere shortening but protects mitochondrial function under oxidative stress. J Cell Sci 121: 1046-1053, 2008.

40. Wan T, Weir EJ, Johnson M, Korolchuk VI and Saretzki GC: Increased telomerase improves motor function and alpha-synuclein pathology in a transgenic mouse model of Parkinson's disease associated with enhanced autophagy. Prog Neurobiol 199: 101953-101953, 2021.

41. Puttarak P, Dilokthornsakul P, Saokaew S, Dhippayom T, Kongkaew C, Sruamsiri R, Chuthaputti A and Chaiyakunapruk N: Effects of Centella asiatica (L.) Urb. on cognitive function and mood related outcomes: A Systematic Review and Meta-analysis. Sci Rep 7: 10646-10646, 2017.

42. Han QQ, Shen TT, Wang F, Wu PF and Chen JG: Preventive and Therapeutic Potential of Vitamin C in Mental Disorders. Curr Med Sci 38: 1-10, 2018

43. Islam MT, Salehi B, Karampelas O, Sharifi-Rad J, Docea AO, Martorell $\mathrm{M}$ and Calina D: High skin melanin content, vitamin D deficiency and immunity: potential interference for severity of COVID-19. Farmacia 68: 970-983, 2020.

44. Sidiropoulou P, Docea AO, Nikolaou V, Katsarou MS Spandidos DA, Tsatsakis A, Calina D and Drakoulis N: Unraveling the roles of vitamin D status and melanin during Covid-19 (Review). Int J Mol Med 47: 92-100, 2021.

45. Islam MT, Quispe C, Martorell M, Docea AO, Salehi B, Calina D, Reiner Ž and Sharifi-Rad J: Dietary supplements, vitamins and minerals as potential interventions against viruses: Perspectives for COVID-19. Int J Vitam Nutr Res: Jan 13, 2021 (Epub ahead of print).

46. Ragab D, Soliman D, Samaha D and Yassin A: Vitamin D status and its modulatory effect on interferon gamma and interleukin-10 production by peripheral blood mononuclear cells in culture. Cytokine 85: 5-10, 2016.

47. Farahzadi R, Fathi E, Mesbah-Namin SA and Zarghami N: Zinc sulfate contributes to promote telomere length extension via increasing telomerase gene expression, telomerase activity and change in the TERT gene promoter $\mathrm{CpG}$ island methylation status of human adipose-derived mesenchymal stem cells. PLoS One 12: e0188052, 2017.
48. Wei F, Qu C, Song T, Ding G, Fan Z, Liu D, Liu Y, Zhang C, Shi S and Wang S: Vitamin C treatment promotes mesenchymal stem cell sheet formation and tissue regeneration by elevating telomerase activity. J Cell Physiol 227: 3216-3224, 2012.

49. Islam MS, Quispe C, Hossain R, Islam MT, Al-Harrasi A, Al-Rawahi A, Martorell M, Mamurova A, Seilkhan A, Altybaeva N, et al: Neuropharmacological Effects of Quercetin: A Literature-Based Review. Front Pharmacol 12: 665031, 2021.

50. Sharifi-Rad J, Dey A, Koirala N, Shaheen S, El Omari N, Salehi B, Goloshvili T, Cirone Silva NC, Bouyahya A, Vitalini S, et al: Cinnamomum Species: Bridging Phytochemistry Knowledge, Pharmacological Properties and Toxicological Safety for Health Benefits. Front Pharmacol 12: 600139-600139, 2021.

51. Zarini GG, McLean M, Vaccaro J, Exebio J, Ajabshir S and Huffman FG: Effect of Vitamin D3 Supplementation on Telomerase Activity in Hispanics with Type 2 Diabetes. FASEB J 30: 1156.1-1156.1, 2016.

52. Salehi B, Calina D, Docea AO, Koirala N, Aryal S, Lombardo D, Pasqua L, Taheri Y, Marina Salgado Castillo C, Martorell M, et al: Curcumin's Nanomedicine Formulations for Therapeutic Application in Neurological Diseases. J Clin Med 9: $35,2020$.

53. Deshpande P, Gogia N and Singh A: Exploring the efficacy of natural products in alleviating Alzheimer's disease. Neural Regen Res 14: 1321-1329, 2019.

54. Sharifi-Rad M, Anil Kumar NV, Zucca P, Varoni EM, Dini L, Panzarini E, Rajkovic J, Tsouh Fokou PV, Azzini E, Peluso I, et al: Lifestyle, Oxidative Stress, and Antioxidants: Back and Forth in the Pathophysiology of Chronic Diseases. Front Physiol 11: 694, 2020.

55. Gupta C and Prakash D: Nutraceuticals for geriatrics. J Tradit Complement Med 5: 5-14, 2014.

56. Sharifi-Rad J, Quispe C, Herrera-Bravo J, Martorell M, Sharopov F, Tumer TB, Kurt B, Lankatillake C, Docea AO, Moreira AC, et al: A Pharmacological Perspective on Plant-derived Bioactive Molecules for Epilepsy. Neurochem Res 46: 2205-2225, 2021.

57. Wyss-Coray T: Ageing, neurodegeneration and brain rejuvenation. Nature 539: 180-186, 2016.

58. Guan JZ, Guan WP, Maeda T, Guoqing X, GuangZhi W and Makino N: Patients with multiple sclerosis show increased oxidative stress markers and somatic telomere length shortening. Mol Cell Biochem 400: 183-187, 2015.

59. Guo T, Zhang D, Zeng Y, Huang TY, Xu H and Zhao Y: Molecular and cellular mechanisms underlying the pathogenesis of Alzheimer's disease. Mol Neurodegener 15: 40-40, 2020.

60. Apostolova $\mathrm{N}$ and Victor VM: Molecular strategies for targeting antioxidants to mitochondria: Therapeutic implications. Antioxid Redox Signal 22: 686-729, 2015.

This work is licensed under a Creative Common Attribution-NonCommercial-NoDerivatives 4.0 International (CC BY-NC-ND 4.0) License. 